\title{
The Analysis of Depolarization Factors in the Last RHIC Run
}

\author{
V. Ptitsyn*, A. U. Luccio* and V. H. Ranjbar ${ }^{\dagger}$ \\ ${ }^{*}$ Brookhaven National Laboratory, Upton NY 11973 \\ ${ }^{\dagger}$ Indiana University, Bloomington IN 47405
}

\begin{abstract}
Polarized proton beams were accelerated succesfully at RHIC up to $100 \mathrm{Gev}$ with the use of Siberian Snakes. Although the snakes were designed to preserve polarization, the succesful acceleration and storage of polarized beams was dependent also on beam characteristics, like closed orbit, betatron tunes and even betatron coupling. The high-order spin resonances were observed and evaluated. The paper summarizes depolarizing effects observed during the run.
\end{abstract}

\section{INTRODUCTION}

At the last run polarized protons were accelerated in both RHIC rings up to $100 \mathrm{GeV}$ energy $(G \gamma=192)$ where they were used for colliding beam experiments [1]. The injection energy for polarized beams was at $24.3 \mathrm{Gev}(G \gamma=46.5)$. The polarization preservation during the beam acceleration from the injection to the top energy was provided by means of Siberian Snakes installed into the RHIC rings (two full snakes in each ring).

Although the Snakes succesfully accomplished their task, the polarization preservation was not always perfect. On some acceleration ramps the significant depolarization was observed. It was noted that the polarization preservation efficiency was sensitive to the beam conditions like closed orbit, betatron tune and betatron coupling.

Although, the Siberian Snakes help to avoid first-order imperfection and intrinsic spin resonances, higher-order spin resonances can occur at the so-called snake resonance conditions [2]:

$$
\delta v_{z}=\frac{v_{s p} \pm k}{l}
$$

where $\delta v_{z}$ is fractional part of vertical betatron tune, and with the Snake setup at RHIC $v_{s p}=1 / 2$.

Additional spin resonances might be caused by the betatron coupling effect. Coupling originates from quadrupole roll errors or solenoidal fields in the accelerator. This betatron coupling introduces the frequency of the horizontal betatron motion into vertical oscillations which leads to "coupled" snake resonances with conditions:

$$
\delta v_{x}=\frac{v_{s p} \pm k}{l}
$$

Vertical closed orbit errors are responsible for the generation of even order snake resonances (with $l$ even in (1) and (2)). It has been shown [2] that in the presence of 


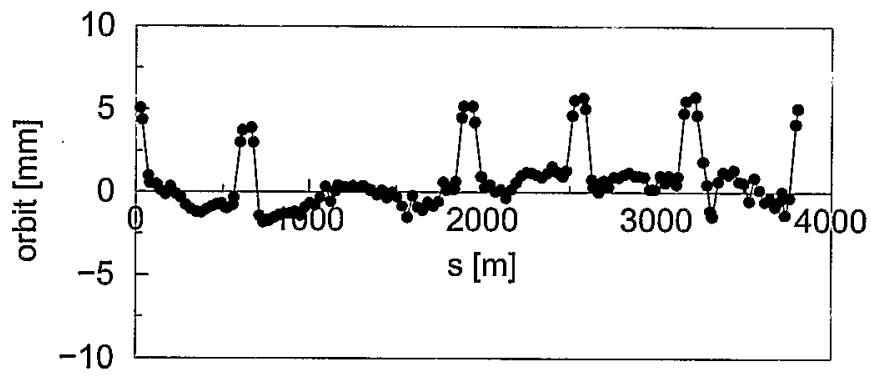

FIGURE 1. Ideal vertical orbit for polarized protons in Blue ring, as seen at Beam Position Monitors. The $5 \mathrm{~mm}$ orbit bumps were introduced in interaction regions to prevent beam collisions during the acceleration. Their depolarization effect is small.

closed orbit errors each snake resonance will split into two, separated by

$$
\delta v_{z} \leq\left|\frac{1}{\pi l} \arcsin \left[\sin ^{2} \frac{\pi \varepsilon_{i m p}}{N_{s}}\right]\right|
$$

where $\varepsilon_{i m p}$ is the imperfection resonance strength.

\section{MACHINE SETUP}

The major factors determining the beam depolarization were closed orbit errors, betatron tune values and betatron coupling.

The depolarization effect of the vertical closed orbit errors is proportional to $z^{\prime \prime}$ which in fact describes radial fields on the beam orbit. The orbit should be perfectly flat, $z^{\prime \prime}=0$, in order to eliminate the imperfection resonances. Ideal orbit correction, using ideally aligned system of Beam Position Monitors (BPM), can realize this task. But in a real accelerator there are two kinds of errors that prevent a perfect orbit correction. One of them is BPM misalignments relative to quadrupole centers, which in case of the RHIC collider has rms value of about $150 \mu \mathrm{m}$. Another possible error is quadrupole vertical misalignments from some ideal plane, which are caused by ground motion as well as limited alignment precision. In the case of RHIC the quadrupole misalignment errors are dominant, with about $0.9 \mathrm{~mm}$ rms value of quad misalignments over the ring azimuth. In order to compensate for the quadrupole alignment errors a special closed orbit through the BPMs was created based on quadrupole misalignment data. Fig. 1 shows the orbit used as ideal orbit for polarized beam acceleration. Although the orbit does not look flat as measured by BPMs, it should be flat, in reality, assuming that the quadrupole misalignment data are correct. The use of this ideal closed orbit decreases imperfection resonance strength and simplifies the task for the Siberian Snakes.

The precision of orbit correction was limited by triplet gradient errors, but typically was kept below $0.8 \mathrm{~mm}$ as shown at the left plot in Fig. 2 .

The fractional betatron tune space, in which RHIC was operated ranged from 0.20 to 0.25 . The vertical tune was placed at 0.23 , between snake resonances $3 / 14$ and $1 / 4$. 

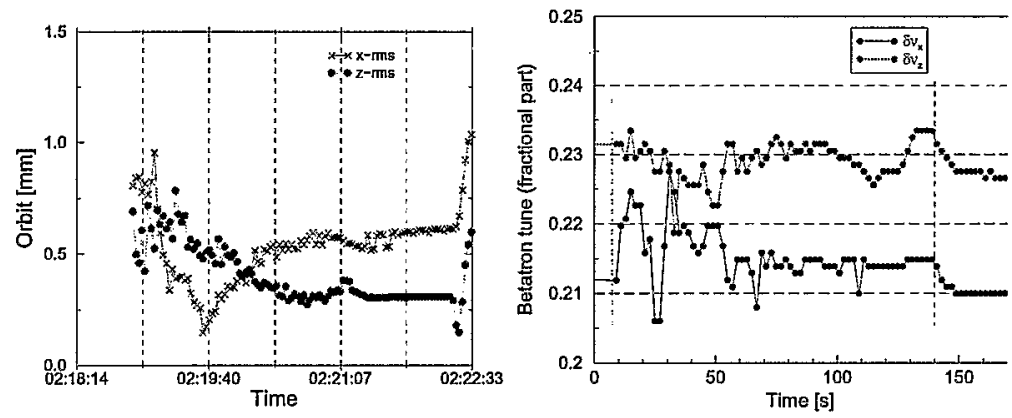

FIGURE 2. The examples for closed orbit and betatron tunes on the ramp. Left plot demonstrates the closed orbit horizontal and vertical rms value during the acceleration. Right plot shows horizontal and . vertical betatron tunes during the acceleration.

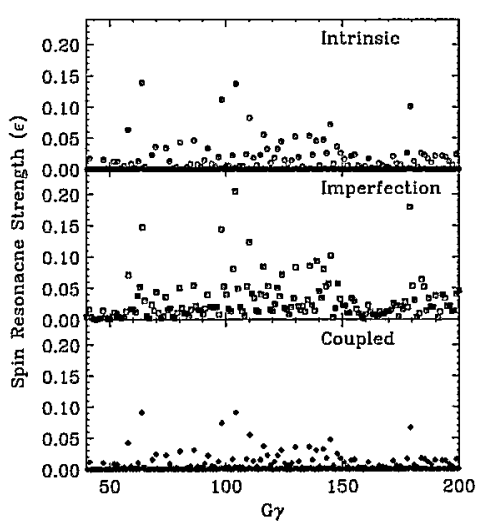

FIGURE 3. Calculated imperfection, intrinsic, and coupled spin resonance strength for RHIC $z_{r m s}=2.2$ $\mathrm{mm}$, using vertical and horizontal emittance of $10 \pi \mathrm{mm}-\mathrm{mrad}$.

During the ramp the tunes did not stay constant. An example of tune excursion along the ramp is shown at the right plot in Fig. 2. In some regions, mainly at the first half of the ramp, the tunes approached too close together $(\leq 0.008)$ so that the betatron coupling effect was clearly seen. Betatron coupling was well corrected at the injection and collision energies [3], but was not very well controlled during the acceleration.

The intrinsic, coupled and imperfection resonance strength is presented in Fig. 3 as calculated by new version of DEPOL code [4]. The maximum imperfection resonance strength $\varepsilon_{\text {imp }}<0.2$ determines the maximum tune splitting using the Eq. (3). Thus the maximal incursion of $1 / 4$ snake resonances into the operating tune box might be as large as 0.242 . 

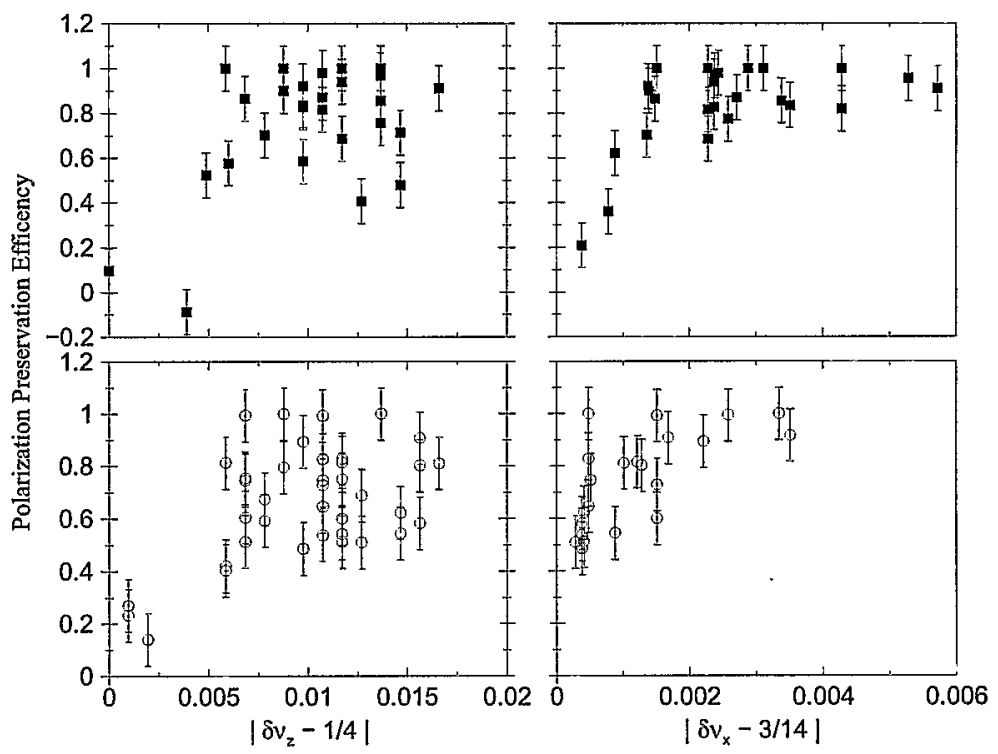

FIGURE 4. $\left|\delta v_{z}-1 / 4\right|$ and $\left|\delta v_{x}-3 / 14\right|$ versus polarization preservation efficiency $\left(P_{f} / P_{i}\right)$ shown for the Yellow (square symbol) and Blue (open circles) rings. In the case of $\left|\delta v_{z}-1 / 4\right|$, polarization preservation efficiency was scattered between 1.0 and 0.4 . This is because other parameters fluctuated each fill.

\section{OBSERVED RESONANCES}

The depolarization caused by two high-order spin resonances, $\delta v_{x}=3 / 14$ and $\delta v_{z}=$ $1 / 4$, was observed during the run and clearly identified from the analysis of the polarization transfer efficiency data. The results of the analysis are shown in Fig. 4.

The right graphs of Fig. 4 show the polarization preservation efficiency for the $3 / 14$ coupled snake resonance versus the resonance proximity parameter, $\left|\delta v_{x}-3 / 14\right|$ for both Blue and Yellow rings. The observed resonance width is defined by the value of the betatron coupling and by resonance splitting effect described by Eq. (3). A larger resonance width was observed in Yellow ring which is consistent with the fact that the Yellow ring had usually larger values of both betatron coupling and closed orbit errors.

On several occasions the vertical tune did approach $1 / 4$ which allowed us to see the depolarization from the $1 / 4$ snake resonance. The left graphs of Fig. 4 clearly show the onset of the $1 / 4$ snake resonance. The width of the resonance is defined by the closed orbit errors. From these graphs the estimated imperfection resonance strength is about 0.16 for both rings. This agrees well with DEPOL calculations.

The depolarization from coupled snake resonance $\delta v_{x}=3 / 14$ was confirmed by spin tracking studies. The tracking results in the Blue ring using the program SPINK [5] with betatron coupling effect included show clearly the depolarization as the horizontal tune crosses the $3 / 14$ snake resonance location in Fig. 5 . 


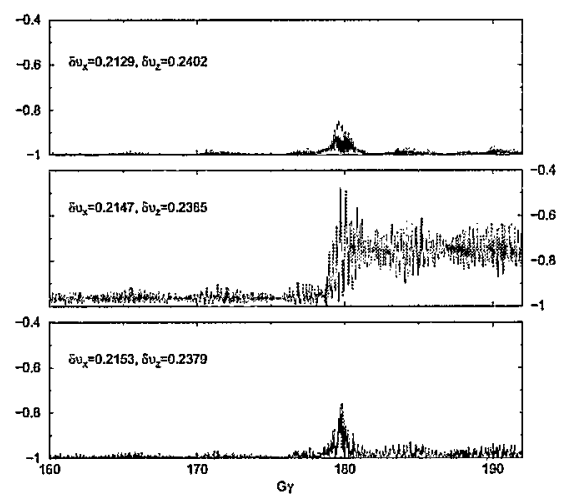

FIGURE 5. Spin Tracking results for strongly coupled Blue ring with an emittance of $25 \mathrm{~mm}$-mrad and $z_{r m s}=0.6 \mathrm{~mm}$. The three graphs show polarization versus $G \gamma$ with the fractional part of the horizontal betatron tunes near $3 / 14$.

\section{CONCLUSION}

The depolarization effects from high-order spin resonances were observed during the polarized proton run at RHIC. The resonance, $\delta v_{x}=3 / 14$, was caused by the betatron coupling. Another resonance, $\delta v_{z}=1 / 4$, was due to the closed orbit errors.

For the next polarized proton run several improvements should help to avoid the depolarization. For better tune control the PLL feedback system will be applied. The new quadrupole misalignment data should provide a more accurate ideal orbit. With the elimination of gradient errors in the IR triplets, the quality of the closed orbit correction is expected to improve below $0.5 \mathrm{~mm}$ rms level. The techniques to measure and correct the betatron coupling on the acceleration ramp are under discussions.

\section{ACKNOWLEDGEMENTS}

The authors would like to thank H. Huang, S.Y. Lee, W.W. MacKay, T. Roser, and S. Tepikian for discussions of various aspects of depolarization analysis. We are also grateful to the RHIC polarimetry team. U.S. Department of Energy.

\section{REFERENCES}

1. H.Huang, "Acceleration of Polarized Protons at RHIC", these proceedings.

2. S. Y. Lee and S. Tepikian, Phys. Rev. Lett. 56, 1635 (1986); S. Tepikian, Ph. D. thesis, State University of New York at Stony Brook, (1986) (unpublished).

3. F. Pilat, et. al, Proc. of EPAC2002, Paris, p. 1178.

4. E. D. Courant and R.D. Ruth, BNL 51270 (1980), (unpublished).; V. Ranjbar et. al., Proc. of EPAC2002, Paris, p. 359.

5. A.U.Luccio Trends in Collider Spin Physics World Scientific, p. 235 (1997). 